\title{
Theoretical analysis on stress distribution characteristics around a shallow buried spherical Karst cave containing fill materials in limestone strata
}

\author{
Peng Xie (D 526458865@qq.com ) \\ Hainan University \\ Shaokun Ma ( $\sim 261688417 @ q q . c o m$ ) \\ Guangxi University \\ Haijia Wen ( $\sim$ jhw@cqu.edu.cn ) \\ Chongqing University \\ Liangyong $\mathrm{Li}$ \\ Hainan University
}

\section{Research Article}

Keywords: Shallow buried, Spherical Karst cave, Spatial stress distribution characteristics in limestone strata, Love displacement function, Theoretical analysis

Posted Date: June 18th, 2021

DOI: https://doi.org/10.21203/rs.3.rs-164261/v1

License: (c) (i) This work is licensed under a Creative Commons Attribution 4.0 International License.

Read Full License

Version of Record: A version of this preprint was published at Environmental Earth Sciences on January 31st, 2022. See the published version at https://doi.org/10.1007/s12665-021-10112-y. 
1 Theoretical analysis on stress distribution characteristics around a

2 shallow buried spherical Karst cave containing fill materials in

3 limestone strata

4 Peng Xie ${ }^{1}$, Shaokun Ma ${ }^{2 *}$, Haijia Wen ${ }^{3,4 *}$, Liangyong $\mathrm{Li}^{1}$

5 First author:

6 Peng Xie E-mail: Peng_Xie@hainanu.edu.cn

7 Corresponding author:

$8 \quad$ 1. Shaokun Ma E-mail: 261688417@qq.com

$9 \quad 2.2 . H a i j i a$ Wen E-mail: jhw@cqu.edu.cn

${ }^{1}$ College of Civil Engineering and Architecture, Hainan University, Haikou, Hainan 570228, China

${ }^{2}$ Guangxi Key Laboratory of Disaster Prevention and Engineering Safety, Guangxi University, Nanning,

Guangxi 530004, China

${ }^{3}$ Key Laboratory of New Technology for Construction of Cities in Mountain Area (Chongqing University),

Ministry of Education, Chongqing 400045, China

${ }^{4}$ School of Civil Engineering, Chongqing University, Chongqing 400045, China

\begin{abstract}
The prime objective of the article is to present the spatial characteristics of stress distribution around a shallowly buried spherical Karst cave containing fill materials in limestone strata. Firstly, considering the external load effects, stress field in the Earth's crust, internal filling, and the Karst landform characteristics in China, a spatial axial-symmetrical model was established. Concurrently, combining available work and the concept of elasticity, the boundary conditions were determined. Subsequently, Love displacement method was introduced, in addition to the expressions of stress components were gained. The diagram characteristics of each stress component were summarized, which are affected by various influencing factors. Finally, in order to prove the rationality of the general solution, a comparison between numerical simulation result and theoretical calculation result was carried out, as well as the maximum error between theoretical calculation value and numerical simulation value is less than $5 \%$. Thus, the analytical solution could represent the spatial characteristics of stress distribution around a shallowly buried spherical Karst cave containing fill materials in limestone strata.
\end{abstract}

Keywords: Shallow buried; Spherical Karst cave; Spatial stress distribution characteristics in limestone strata; Love displacement function; Theoretical analysis

\title{
1. Introduction
}

Karst landform exists widely in the world, and Karst cave is one of the typical representatives. 
Up to now, various patterns of Karst cave have been found, including columnar, alter shaped, spherical, funnel shaped and so on [1,2], and filled by fill materials (such as clay, water, air and so on). Different from abroad [3], most of this area is shallowly buried. In recent years, owing to the rapid urban development as well as the growing occupation of terrestrial, the dimensions as well as the speed of engineering construction have been greatly accelerated [4]. The ground collapse has become a common engineering problem in mantled Karst region, and brings great loss to people's life and property. The losses encompass agricultural engineering, highway engineering, railway engineering, mining engineering, industrial and civil construction engineering, etc [5-8]. Therefore, the prevention and control of ground collapse is an important requirement for national security and economic development.

Generally, carbonate rocks are dissolved by faintly acidic waters [9-11], Karst caves may provide transport channel or storage space for overlying rock/soil mass, and cause changes in spatial stress distribution. To investigate the effect on spatial stress distribution ,caused by buried Karst caves, a lot of research was performed. Goodier [12] presented that there is a concentration around void or defect. In order to achieve quantitative expression of stress distribution, Howland et al. [13] simplified the problem into a thin plate containing circular holes, and inverse method was used to solved the problem. Taking into account the variety of Karst caves and the complexity of loading conditions, Rao et al. [14] analysed the stresses of surrounding rock containing a tubular filled elliptical karst cave, and the analytic general formula for the component of stresse were solved. Li et al. [15] established the plane mechanical models with different stress boundary conditions in each direction, and the exact general solution was obtained. Shi et al. [16] summarized the stress distribution around rectangular cavity. Considering the spatial geometric characteristics of Karst caves, Liao et al. [17] provided the extreme value of critical point on the wall of an elliptical spherical cavity under triaxial stress.

To sum up, a large number of studies have been conducted. However, spatial geometry of strata and internal filling are limited, and the general solution of stress distribution around Karst cave was not provided in the research process. In addition, complex function theory was mainly used, the method is relatively single and the function is relatively complex. Therefore, Love displacement was induced to study the spatial stress distribution characteristics around a shallowly buried spherical Karst cave containing fill materials in limestone strata. Firstly, considering the geometry of limestone formations, a spatial axial-symmetrical model was established. Concurrently, on the basis of the theory of elasticity, the expressions of stress components were obtained. Finally, a numerical simulation was carried out to prove the rationality of the general solution.

\section{Mechanical model and boundary conditions}

\subsection{Mechanical model}

According to the characteristic of Karst landform in China, Karst caves are usually affected by internal filling, external load and stress field in the Earths crust (Figure 1). In order to facilitate analytical analysis, the basic assumptions are as follows: (1) the limestone strata can be simplified into a spatial axial-symmetrical model. (2) the spherical Karst cave containing fill materials are shallowly buried in limestone strata $(h<2.5 D$, the hidden depth of the Karst cave is $h$, the diameter of the karst cave is $D$ ). (3) The limestone strata is homogeneous, continuous and isotropic. In addition, the parameters are $\gamma$ (unit weight), $\mu$ (poisson's ratio) and $E$ (elastic modulus) respectively. 
Based on above assumptions, the spherical coordinates $(r, \theta, \varphi)$ is chosen as the coordinate system (Figure 2). The effect generated by external loads and the gravity of overlying limestone are simplified into vertical uniform distributed loads. In addition, stress field in the Earths crust will cause vertical stress on the bottom and horizontal stress surrounding the Karst cave (the side force coefficient $\left.k_{0}=\mu /(1-\mu)\right)$.

The parameters in Figure 1 and Figure 2 are

$p_{z}-$ external vertical load;

$p_{0}-$ horizontal stress caused by external vertical load and field in the Earths crust, $p_{0}=$ $k_{0}\left[p_{z}+\chi(h+z)\right]$

$p_{\mathrm{i}}$ _ radial stress caused by fill materials;

$h$ - The vertical distance from the top of the limestone strata to the center of the spherical Karst cave;

$R$ — the radius of the buried spherical karst cave;

$k_{0}$ _ the side force coefficient, $k_{0}=\mu /(1-\mu)$.

\subsection{Boundary conditions}

Combining available work and the concept of elasticity, the boundary conditions are

(1) $z=-h, \sigma_{z}=p_{z}$ is the vertical stress generated by external vertical load;

(2) $r \rightarrow \infty, \quad \sigma_{r}=\left[p_{z}+\gamma(h+z)\right] \mu /(1-\mu)$ is the radial stress considering external vertical load and the gravity of the medium of the strata;

(3) $r \rightarrow \infty, \sigma_{\theta}=\left[p_{z}+\gamma(h+z)\right] \mu /(1-\mu)$ is the tangential stress considering external vertical load and and the gravity of the medium of the strata;

(4) $R=R_{1}, \sigma_{R}=p_{i}$ is the internal pressure considering the gravity of fill materials of the buried spherical karst cave .

\section{Theoretical analysis}

\subsection{The basic theory [18-19]}

Love displacement method was induced in this paper, which is an effective way to solve spatial axial-symmetrical problem.

Taking into account the influence of gravity, the equilibrium differential equations become as

$$
\left.\begin{array}{c}
\frac{\partial \sigma_{r}}{\partial r}+\frac{\partial \tau_{z r}}{\partial z}+\frac{\sigma_{r}-\sigma_{\theta}}{r}=0 \\
\frac{\partial \sigma_{z}}{\partial z}+\frac{\partial \tau_{z r}}{\partial r}+\frac{\tau_{z r}}{r}+\gamma=0
\end{array}\right\}
$$

In addition, the stress components are as follows, using Love displacement function.

$$
\left.\begin{array}{l}
\sigma_{r}=\frac{\partial}{\partial z}\left(\mu \nabla^{2}-\frac{\partial^{2}}{\partial r^{2}}\right) \varphi(r, z) \\
\sigma_{\theta}=\frac{\partial}{\partial z}\left(\mu \nabla^{2}-\frac{1}{r} \frac{\partial}{\partial r}\right) \varphi(r, z) \\
\sigma_{z}=\frac{\partial}{\partial z}\left[(2-\mu) \nabla^{2}-\frac{\partial^{2}}{\partial z^{2}}\right] \varphi(r, z) \\
\tau_{r z}=\frac{\partial}{\partial r}\left[(1-\mu) \nabla^{2}-\frac{\partial^{2}}{\partial z^{2}}\right] \varphi(r, z)
\end{array}\right\}
$$


The spherical coordinates $(r, \theta, \varphi)$ is chosen as the coordinate system,the relationship of the stress components between spherical coordinates and cylindrical coordinates are as follows

$$
\left.\begin{array}{l}
\sigma_{R}=\sigma_{r} \sin ^{2} \varphi+\sigma_{z} \cos ^{2} \varphi+2 \tau_{r z} \sin \varphi \cos \varphi \\
\sigma_{\theta}=\sigma_{\theta} \\
\sigma_{\varphi}=\sigma_{r} \cos ^{2} \varphi+\sigma_{z} \sin ^{2} \varphi-2 \tau_{r z} \sin \varphi \cos \varphi \\
\tau_{R \varphi}=\left(\sigma_{r}-\sigma_{z}\right) \sin \varphi \cos \varphi-\tau_{r z}\left(\sin ^{2} \varphi-\cos ^{2} \varphi\right)
\end{array}\right\}
$$

\subsection{The general solution}

To obtain the general solution of stress components using Love displacement method mainly includes three parts: Established the Love displacement function, the undetermined coefficients of each stress components expressions were solved, and the result was processed (Figure 3).

In this study, spherical coordinate system was selected as the coordinate system. Love displacement function (Equation (2)) was established, which meets Equation (1) [18-22].

$$
\varphi=A_{2} r^{4}+A_{3} z^{3}+A_{4} z^{2} r^{2}+A_{5} z r^{2}+A_{9} z R^{-1}
$$

where, $A_{i}(i=2,3,4,5,9)$ are the undetermined coefficients

Substituting Equation (4) into Equation (2) yields

$$
\sigma_{r}=4\left[(2 \mu-1) A_{4}\right] z+2\left[3 \mu A_{3}+(2 \mu-1) A_{5}\right]+A_{9}\left[15 r^{2} z^{2} R^{-7}+3(2 \mu-1) z^{2} R^{-5}-3 r^{2} R^{-5}+(1-2 \mu) R^{-3}\right]
$$

$$
\begin{aligned}
& \sigma_{\theta}=4\left[(2 \mu-1) A_{4}\right] z+2\left[3 \mu A_{3}+(2 \mu-1) A_{5}\right]+A_{9}\left[3(2 \mu-1) z^{2} R^{-5}+(1-2 \mu) R^{-3}\right. \\
& \sigma_{z}=8\left[(2-\mu) A_{4}\right] z+2\left[3(1-\mu) A_{3}+2(2-\mu) A_{5}\right]+A_{9}\left[15 z^{4} R^{-7}-6(1+\mu) z^{2} R^{-5}+(2 \mu-1) R^{-3}\right] \\
& \tau_{r z}=4\left[8(1-\mu) A_{2}-\mu A_{4}\right] r+A_{9}\left[15 r z^{3} R^{-7}-3(1+2 \mu) r z R^{-5}\right]
\end{aligned}
$$

Substituting the expressions of the stress components into Equation (3) yields $\sigma_{R}=\left\{4\left[16(1-\mu) A_{2}-A_{4}\right] \sin ^{2} \varphi \cos \varphi+8\left[(2-\mu) A_{4}\right] \cos ^{3} \varphi\right\} R+\left\{2\left[3 \mu A_{3}+(2 \mu-1) A_{5}\right] \sin ^{2} \varphi+2[3(1\right.$ $\left.\left.-\mu) A_{3}+2(2-\mu) A_{5}\right] \cos ^{2} \varphi\right\}+A_{9}\left[15 \cos ^{6} \varphi+15 \sin ^{4} \varphi \cos ^{2} \varphi+30 \sin ^{2} \varphi \cos ^{4} \varphi-3 \sin ^{4} \varphi-6(1\right.$ $\left.+\mu) \cos ^{4} \varphi-3(3+2 \mu) \sin ^{2} \varphi \cos ^{2} \varphi+(1-2 \mu) \sin ^{2} \varphi+(2 \mu-1) \cos ^{2} \varphi\right] \frac{1}{R^{3}}$

$$
\sigma_{\theta}=\left\{4\left[(2 \mu-1) A_{4}\right] \cos \varphi\right\} R+2\left[3 \mu A_{3}+(2 \mu-1) A_{5}\right] A_{9}\left[3(2 \mu-1) \cos ^{2} \varphi+(1-2 \mu)\right] \frac{1}{R^{3}}
$$

$\sigma_{\varphi}=\left\{4\left[(2 \mu-1) A_{4} \cos ^{3} \varphi+8\left[-8(1-\mu) A_{2}+2 A_{4}\right] \sin ^{2} \varphi \cos \varphi\right\} R+\left\{2\left[3 \mu A_{3}+(2 \mu-1) A_{5}\right] \cos ^{2} \varphi+2[3(1\right.\right.$ 


$$
\begin{aligned}
& \left.\left.-\mu) A_{3}+2(2-\mu) A_{5}\right] \sin ^{2} \varphi\right\}+A_{9}\left[3(2 \mu-1) \cos ^{2} \varphi+(1-2 \mu)\left(\cos ^{2} \varphi-\sin ^{2} \varphi\right)\right] \frac{1}{R^{3}} \\
\tau_{R \varphi}= & \left\{4\left[8(1-\mu) A_{2}+(3 \mu-5) A_{4}\right] \sin ^{2} \varphi \cos ^{2} \varphi-4\left[8(1-\mu) A_{2}-\mu A_{4}\right] \sin ^{3} \varphi\right\} R+\left[6(2 \mu-1) A_{3}+2(4 \mu-5\right. \\
& \left.A_{5}\right] \sin \varphi \cos \varphi+2 A_{9}(1+\mu) \frac{1}{R^{3}} \sin \varphi \cos \varphi
\end{aligned}
$$

Equation (9)-(12) are proved to satisfy Equation (1), so

$$
8 A_{2}+2 A_{4}=\frac{\gamma}{8(\mu-1)}
$$

Substituting boundary condition (1) into Equation (7)

$-8\left[(2-\mu) A_{4}\right] h+2\left[3(1-\mu) A_{3}+2(2-\mu) A_{5}\right]-A_{9} \frac{1}{h^{3}}\left[15 \cos ^{7} \varphi-6(1+\mu) \cos ^{5} \varphi+(2 \mu-1) \cos ^{3} \varphi\right]=p_{z}$

Substituting boundary condition (2), (3) into Equation(5), (6) respectively, and matching the equivalent coefficients of both sides leads to

$$
\begin{gathered}
4(2 \mu-1) A_{4}=\frac{\mu}{1-\mu} \gamma \\
6 \mu A_{3}+2(2 \mu-1) A_{5}=\frac{\mu}{1-\mu}\left(p_{z}+\gamma h\right)
\end{gathered}
$$

Substituting boundary condition (4) into Equation (9)

$\left\{4\left[16(1-\mu) A_{2}-A_{4}\right] \sin ^{2} \varphi \cos \varphi+8\left[(2-\mu) A_{4}\right] \cos ^{3} \varphi\right\} R_{1}+\left\{2\left[3 \mu A_{3}+(2 \mu-1) A_{5}\right] \sin ^{2} \varphi+2\left[3(1-\mu) A_{3}\right.\right.$ $\left.\left.+(2-\mu) A_{5}\right] \cos ^{2} \varphi\right\}+A_{9}\left[15 \cos ^{6} \varphi+15 \sin ^{4} \varphi \cos ^{2} \varphi+30 \sin ^{2} \varphi \cos ^{4} \varphi-3 \sin ^{4} \varphi-6(1+\mu) \cos ^{4} \varphi-3(\right.$ $\left.3+2 \mu) \sin ^{2} \varphi \cos ^{2} \varphi+(1-2 \mu) \sin ^{2} \varphi+(2 \mu-1) \cos ^{2} \varphi\right] \frac{1}{R_{1}^{3}}=p_{i}$

Combining Equation (13)-(17), the equations of the stress components are as follows

$\sigma_{R}=\left\{4\left[16(1-\mu) A_{2}-A_{4}\right] \sin ^{2} \varphi \cos \varphi+8\left[(2-\mu) A_{4}\right] \cos ^{3} \varphi\right\} R+\left\{2\left[3 \mu A_{3}+(2 \mu-1) A_{5}\right] \sin ^{2} \varphi+2[3(1-\right.$ $\left.\left.\mu) A_{3}+2(2-\mu) A_{5}\right] \cos ^{2} \varphi\right\}+A_{9}\left[15 \cos ^{6} \varphi+15 \sin ^{4} \varphi \cos ^{2} \varphi+30 \sin ^{2} \varphi \cos ^{4} \varphi-3 \sin ^{4} \varphi-6(1+\right.$ $\left.\mu) \cos ^{4} \varphi-3(3+2 \mu) \sin ^{2} \varphi \cos ^{2} \varphi+(1-2 \mu) \sin ^{2} \varphi+(2 \mu-1) \cos ^{2} \varphi\right] \frac{1}{R^{3}}$

$$
\sigma_{\theta}=\left\{4\left[(2 \mu-1) A_{4}\right] \cos \varphi\right\} R+2\left[3 \mu A_{3}+(2 \mu-1) A_{5}\right]+A_{9}\left[3(2 \mu-1) \cos ^{2} \varphi+(1-2 \mu)\right] \frac{1}{R^{3}}
$$

$\sigma_{\varphi}=\left\{4\left[(2 \mu-1) A_{4} \cos ^{3} \varphi+8\left[-8(1-\mu) A_{2}+2 A_{4}\right] \sin ^{2} \varphi \cos \varphi\right\} R+\left\{2\left[3 \mu A_{3}+(2 \mu-1) A_{5}\right] \cos ^{2} \varphi+2[3(1\right.\right.$

$$
\left.\left.-\mu) A_{3}+2(2-\mu) A_{5}\right] \sin ^{2} \varphi\right\}+A_{9}\left[3(2 \mu-1) \cos ^{2} \varphi+(1-2 \mu)\left(\cos ^{2} \varphi-\sin ^{2} \varphi\right)\right] \frac{1}{R^{3}}
$$


$\tau_{R \varphi}=\left\{4\left[8(1-\mu) A_{2}+(3 \mu-5) A_{4}\right] \sin ^{2} \varphi \cos ^{2} \varphi-4\left[8(1-\mu) A_{2}-\mu A_{4}\right] \sin ^{3} \varphi\right\} R+\left[6(2 \mu-1) A_{3}+2(4 \mu-5\right.$

$$
\left.A_{5}\right] \sin \varphi \cos \varphi+2 A_{9}(1+\mu) \frac{1}{R^{3}} \sin \varphi \cos \varphi
$$

155 Where

$$
A_{2}=\frac{(6 \mu-1)}{64(1-\mu)(1-2 \mu)} \gamma, A_{3}=-\frac{B_{1}}{6 B_{2}}, \quad A_{4}=\frac{\mu \gamma}{4(1-\mu)(2 \mu-1)}, A_{5}=-\frac{B_{3}}{2 B_{2}}, A_{9}=-\frac{B_{4}}{B_{2}}
$$

$B_{1}=2(2 \mu-1) h^{3}\left[(1-\mu)(2 \mu-1) p_{z}+2 \mu(2-\mu) \cos ^{2} \varphi\right]-\mu(2 \mu-1) R_{1}^{3}\left(p_{z}+\gamma h\right)\left[2(2-\mu) \cos ^{2}\right.$ $\left.\varphi+(2 \mu-1) \sin ^{2} \varphi\right]\left[15 \cos ^{7} \varphi-6(1+\mu) \cos ^{5} \varphi+(2 \mu-1) \cos ^{3} \varphi\right]-4 \mu h^{3}(2-\mu)(2 \mu-1)\left(p_{z}+\right.$ hh) $\left[2(2-\mu) \cos ^{2} \varphi-(1+\mu) \sin ^{2} \varphi\right]+(2 \mu-1) R_{1}^{3}\left[15 \cos ^{7} \varphi-6(1+\mu) \cos ^{5} \varphi+(2 \mu-1) \cos ^{3} \varphi\right]$

$$
\left[(1-\mu)(2 \mu-1) p_{i}+(6 \mu-1)(1-\mu) \gamma R_{1} \sin ^{2} \varphi \cos \varphi-2(2-\mu) R_{1} \gamma \cos ^{3} \varphi+\mu R_{1} \gamma \sin ^{2} \varphi \cos \varphi\right]
$$

$B_{2}=(1-\mu)(2 \mu-1)\left\{R_{1}^{3}(1-2 \mu)\left[\mu \sin ^{2} \varphi+(1-\mu) \cos ^{2} \varphi\right]\left[15 \cos 7 \varphi-6(1+\mu) \cos ^{5} \varphi+(2 \mu-1) \cos \right.\right.$ $\left.{ }^{3} \varphi\right]+R_{1}^{3} \mu\left[2(2-\mu) \cos ^{2} \varphi+(2 \mu-1) \sin ^{2} \varphi\right]\left[15 \cos ^{7} \varphi-6(1+\mu) \cos ^{5} \varphi+(2 \mu-1) \cos ^{3} \varphi\right]+4$ $\mu h^{3}(2-\mu)\left[2(2-\mu) \cos ^{2} \varphi-(1+\mu) \sin ^{2} \varphi\right]+2 h^{3}(1-\mu)(1-2 \mu)\left[2(2-\mu) \cos ^{2} \varphi-(1+\mu) \sin \right.$ $\left.\left.{ }^{2} \varphi\right]\right\}$

$B_{3}=-2 \mu h^{3}\left[(1-\mu)(2 \mu-1) p_{z}+2 \mu(2-\mu) \gamma h\right]\left[2(2-\mu) \cos ^{2} \varphi-(1+\mu) \sin ^{2} \varphi\right]+\mu(2 \mu-1) R_{1}^{3}\left(p_{z}+\right.$ hh) $\left[\mu \sin ^{2} \varphi+(1-\mu) \cos ^{2} \varphi\right]\left[15 \cos ^{7} \varphi-6(1+\mu) \cos ^{5} \varphi+(2 \mu-1) \cos ^{3} \varphi\right]+2 \mu h^{3}(1-\mu)(2 \mu-1$ $\left(p_{z}+\gamma h\right)\left[2(2-\mu) \cos ^{2} \varphi-(1+\mu) \sin ^{2} \varphi\right]-\mu\left[15 \cos ^{7} \varphi-6(1+\mu) \cos ^{5} \varphi+(2 \mu-1) \cos ^{3} \varphi\right][(1+$

$$
\left.\mu)(2 \mu-1) R_{1}^{3} p_{i}+(6 \mu-1)(1-\mu) R_{1}^{4} \gamma \cos ^{3} \varphi+\mu R_{1}^{4} \gamma \sin ^{2} \varphi \cos \varphi\right]
$$

$B_{4}=h^{3}\left\{(1-\mu) R_{1}^{3}\left[(1-\mu)(2 \mu-1) p_{z}+2 \mu(2-\mu) \gamma h\right]\left[\mu \sin ^{2} \varphi+(1-\mu) \cos ^{2} \varphi\right]+\gamma R_{1}^{3}\left[(1-\mu)\left(2 \mu p_{z}\right.\right.\right.$ $+2 \mu(2-\mu) \gamma h]\left[2(2-\mu) \cos ^{2} \varphi+(2 \mu-1) \sin ^{2} \varphi\right]+2 \mu(2-\mu)(2 \mu-1) R_{1}^{3}\left(p_{z}+\gamma h\right)\left[\mu \sin ^{2}+(1\right.$ $\left.-\mu) \cos ^{2} \varphi\right]-\mu(1-\mu)(2 \mu-1) R_{1}^{3}\left(p_{z}+\gamma h\right)\left[2(2-\mu) \cos ^{2} \varphi+(2 \mu-1) \sin ^{2} \varphi\right]-2 \mu\left(2-R_{1}^{3}\right.$

$$
\left[(1-\mu)(2 \mu-1) p_{i}+(6 \mu-1)(1-\mu) \gamma R_{1} \sin ^{2} \varphi \cos \varphi-2(2-\mu) R_{1} \gamma \cos ^{3} \varphi+\mu R_{1} \gamma \sin ^{2} \varphi \cos \varphi\right.
$$$$
]+(1-\mu)(2 \mu-1) R_{1}^{3}\left[(1-\mu)(2 \mu-1) p_{i}+(6 \mu-1)(1-\mu) \gamma R_{1} \sin ^{2} \varphi \cos \varphi-2(2-\mu) R_{1} \gamma \cos ^{3}\right.
$$ 


$$
\left.\left.\varphi+\mu R_{1} \gamma \sin ^{2} \varphi \cos \varphi\right]\right\}
$$

Then, the general solution of the stress components are obtained, which can consider the common effect of the external load effects, stress field in the Earth's crust, internal filling, and the Karst landform characteristics in China. In general, the value of is definite for a specific site. Therefore, the radius $(R)$ and the angle $(\varphi)$ are two influencing factors for the characteristics of the stress distribution in the strata..

\section{Distribution characteristics of stress component}

\subsection{Distribution characteristics of stress component}

For stress component, there are two influencing factors, one is the radius $(r)$, another is the angle $(\varphi)$. In order to display the distribution characteristics of each stress component, the data are substituted into the expressions of each stress component, as well as the diagram of each stress component are drawn using MATLAB software.

When angle is constant $(\varphi=\pi / 2)$, the curves of circumferential stress and radial stress are non-linear. Nevertheless, the tendency of value alteration is the opposed with the increase of radius value (Figure 4(a)). Figure 4(b) indicates that tangential stress value increases with the increase of radius value, as well as tend a constant value finally. In addition, the relationship between shear stress and radius is linear (Figure 4(c)).

Furthermore, to discuss the effect of angle $(\varphi)$, the curves of each stress component were drawn in Figure $5(R=2 \mathrm{~m})$. Figure 4 shows the curves of each stress component are symmetrical, and $\varphi=\pi / 2$ is the axis of symmetry except shear stress (angle $(\varphi)$ varies from 0 to $2 \pi$ ). In addition the value of shear stress is positive, as well as the other stress component contains positive and negative value.

In summary, the result is agree with previous research[12]. In the meanwhile, The characteristics of spatial stress distribution was presented through above discussion, which will provide scientific evidence for bearing capacity determination of foundation containing shallowly buried Karst cave in the further research.

\subsection{Validation test of general solution}

To verify the validation of the proposed analytical solution, the numerical simulation was conducted. 

along these survey lines. Owing to the diferent attributes of the media it penetrates, a part of the signals emitted is refected by the interface between the various materials. The refected signals are received, magnifed, and digitized by a receiver and then relayed to the mainframe for storage. The signals in the GPR profles are enhanced via data processing, and the higher accuracy and better visual geophysical signatures are represented. Detailed information is provided by images interpretation, including information of the embedded depth, boundary of the karst caves, and the thickness of the soil and interlayer. A spatial database in coordinate system relative to the geological map could be easily positioned. Finally, based on this spatial database, a series of twodimensional geological cross sections are generated, and a spatial model was generated.Considering the model symmetry, a $1 / 2$ geometry model was selected, and the dimension and mesh are shown in Figure 6, constraints were applied to the bottom of the model in the upright and horizontal direction, and the excavation of void is used to simulate the formation of natural karst cave. Horizontal stress surrounding the karst cave is $p_{2}=8.833 .5 z$ ( $z$ is vertical coordinate value), which is caused by stress field in the Earths crust (Figure 7). The red facts were recycled to monitor the horizontal as well as vertical stress (Figure 8) the parameters of the rock and soil materials. The parameters of buried karst cave and thick limestone are shown in Table 1.

Owing to theoretical analysis and numerical simulation were carried in different coordinate system, the monitoring statistics was converted into the standards in spatial coordinates using Equations (22)-(24). Figure 9 presents the monitoring data and calculation value of stress component together. The theoretical calculation value is consistent with monitoring facts, the extreme fault is not more than $5.0 \%$, which meets the proposal standard necessity in practice.

$$
\sigma_{r}=\sigma_{x} \cos ^{2} \theta+\sigma_{y} \sin ^{2} \theta+2 \tau_{x y} \sin \theta \cos \theta
$$

$$
\sigma_{\theta}=\sigma_{x} \sin ^{2} \theta+\sigma_{y} \cos ^{2} \theta-2 \tau_{x y} \sin \theta \cos \theta
$$




\section{Discussion}

In summary, the expressions of stress components are composed of power function, which are beneficial to the application in practice. Nevertheless, there is a fault (the maximum fault is not more than $5.0 \%$ ) for stress component, it's necessary to optimize the analytic solution in the future. On the one hand, increase the type of Love displacement function component may be an effective way. On the other hand, various method could be introduced.

The characteristics of spatial stress distribution could be presented through using the general solution relatively well. In the further research, the formula of foundation bearing capacity calculation in the hidden Karst region could be obtained, combining failure mechanism of ground collapse and the expressions of stress components.

\section{Conclusion}

Taking into account the effect of external load, stress field in the Earths crust, internal filling and the characteristics of Karst landform in China, a spatial axial-symmetrical model was generated. A general solution of stress components was gained using Love displacement method.

For stress component, there are two influencing factors, one is the radius $(R)$, another is the angle $(\varphi)$. When angle is constant, the curves of circumferential stress and radial stress are non-linear. Nevertheless, the tendency of value alteration is the opposed with the increase of radius value.Tangential stress value increases with the increase of radius value, as well as tend a constant value finally. In addition, the curve of shear stress is linear. Furthermore, when radius is constant, the curves of each stress component are symmetrical except shear stress, and $\varphi=\pi / 2$ is the axis of symmetry. In addition the value of shear stress is positive, as well as the other stress component contains positive and negative value. the analytical solution could represent the spatial characteristics of stress distribution around a shallow buried spherical Karst cave in limestone strata.

Based on ground penetrating radar survey and laboratory analysis of rock in Chongqing, a mathematical model was conducted, and the judgement in the middle of monitoring data and calculation value of stress component was carried out. The theoretical calculation value is consistent with monitoring facts, the extreme fault is not more than $5.0 \%$, which meets the proposal standard necessity in practice. As a result, the analytical solution could provide scientific evidence for bearing capacity determination of foundation containing shallowly buried Karst cave 
in the further research.

\section{Declaration of competing interest}

264 The authors declare that they have no known competing financial interests or personal relationships that could have appeared to influence the work reported in this paper.

\section{Acknowledgements}

The authors are grateful to Yanyan Zhang, Jing Hu and Xiaoxuan Zhang for their assistance.

Funding statement

269

The research on which this article is based has been supported by grants: the Systematic Project of Guangxi Key Laboratary of Disaster Prevention Structural Safety (Grant No:2019ZKX015), the Science Foundation of Hebei Province (No. E2020210006), the Scientific Research Startup Foundation of Hainan university (Grant No:KYQD(2R)1723), Key Research and Development Project of Cangzhou Municipal Science and Technology bureau,China(Grant No.183305006), National Key R\&D Program of China (Grant No. 2018YFC1505501).

\section{Authors' Contributions:}

Peng xie carried out theoretical analysis; Shaokun Ma and Haijia Wen carried out numerical simulation; Runbin Li and Runzu Yue carried out on-site investigation; Junfeng Liu, Bingyang Liu and Liangyong Li carried out Data statistics. All authors have give finial approval for publication.

\section{References}

[1] Gutierrez F, Parise M, Dewaele J, Jourde H. A review on natural and human-induced geohazards and impacts in karst[J]. Earth-Science Reviews, 2014, 138: 61-88.

[2] Xie P, Wen HJ, Xiao P, Zhang YY. Evaluation of ground-penetrating radar (GPR) and geology survey for slope stability study in mantled karst region[J]. Environmental Earth Sciences (2018) 77:122.

[3] Zhao H J, Ma F S, Guo J. Regularity and formation mechanism of Large-scale abrupt karst collapse in southern China in the first half of 2010[J]. Nat Hazard, 2012, 60: 1037-1045.

[4] Peng Xie, Haijia Wen, Yanyan Zhang, Xiaoxuan Zhang, Jing Hu. A method for identification and reconstruction of hard structural planes, weak interlayer, and cavities in the limestone near surface[J] European Journal of Environmental and Civil Engineering . DOI: 10.1080/19648189.2018.1512902.

[5] Scotto A S, Forte S, A. Analysis of sinkhole triggering mechanisms in the hinterland of Naples (southern 
[6] Kaufmann G, Romanov D, Tippelt T, Vienken T, Werban U, Dietrich P, Mai F, Börner F. Mapping and modelling of collapse sinkholes in soluble rock: The Münsterdorf site, northern Germany[J]. Journal of Applied Geophysics, 2018,154: 64-80.

[7] Wan D D. The Traders' Cave of Niah (NW Borneo): morphology and features as indicators of speleogenesis and karstification[J]. Carbonates Evaporites, 2018, 33: 315-329.

[8] Harris P, Purkis S, Reyes B. Statistical pattern analysis of surficial karst in the Pleistocene Miamioolite of South Florida[J]. Sedimentary Geology, 2018, 367: 84-95.

[9] Zhang L F, Zeng X S, Yao Y S, Liao W L. Review on karst collapse in China[J].The Chinese Journal of Geological Hazard and Control, 2007, 18(3):126-130. (in Chinese)

[10] Williams P W. The role of the epikarst in karst and cave hydrogeology: a review[J]. Int. J. Speleol, 2008, 37: $1-10$.

[11] Piccini L, Mecchia M. Solution weathering rate and origin of karst landforms and caves in the quartzite of Auyan-tepui (Gran Sabana, Venezuela)[J]. Geomorphology, 2009, 106:15-25.

[12] Goodier J N. Concentrations of stress around spheroidal and cylindrical inclusions and flaws[J]. Journal of Applied Mechanics, 1933, 55: 39-44.

[13] Howland R C J, Knight R C. Stress functions for a plate containing groups of circular holes[J]. Philosophical Transactions of the Royal Society A: Mathematical and Physical Sciences, 1939, 238(2): 357-392.

[14] Rao J Y, Fu H L, Liu Y S, Yin Q. Stress analysis of rocks surrounding a tubular filled elliptical karst cave with complex function of elastic mechanics[J]. Journal of Central South University (Science and Technology), 2015, 46(7):2605-2612. (in Chinese)

[15] Li Q Q, Zhang D L, Fang Q. Analytic solution to initial damage of cavern strata by complex function method[J]. Chinese Journal of Geotechnical Engineering, 2014, 36(11):2110-2117. (in Chinese)

[16] Shi G P, Zhu J H, Li B H, Yang J H. Elastic analysis of hole-edge stress of rectangular roadway[J]. Rock and Soil Mechanics, 2014, 35(9):2587-2593. (in Chinese)

[17] Liao L P, Yang W K, Wang Q Z. Stability analysis of an ellipsoidal cavity in foundation[J]. Rock and Soil

319 [18] A. E. H. Love et al. Mathematical Theory of Elasticity[M]. London: PETTER LANE, E. C. 1920.

[19] Timoshenko, S., Goodier, J. N. Theory of elasticity [M]. Xu Zhilun, WuYongzhen translation. Beijing: Higher Education Press, 1965. 
[20] Liang, Y., Wang, H. \& Ren, X., 2008. Analytical solution for spatially axisymmetric problem of thick-walled cylinder subjected to different linearly varying pressures along the axis and uniform pressures at two ends, Sci. China Ser. G - Phys. Mech. Astron., 51(1), 98-104.

[21] Peng Xie , Haijia Wen, Guijun Wang. An analytical solution of stress distribution around underground gas storage cavern in bedded salt rock[J]. Journal of Renewable and Sustainable Energy, 2018, 10(3): 034101.

[22] Peng Xie, Haijia Wen, Guijun Wang, Jing Hu. Theoretical analytical solution of deformation and stress distribution of underground gas storage cavern in bedded salt rock. Archives of Civil Engineering, 2018, LXIV(4): 37-53.

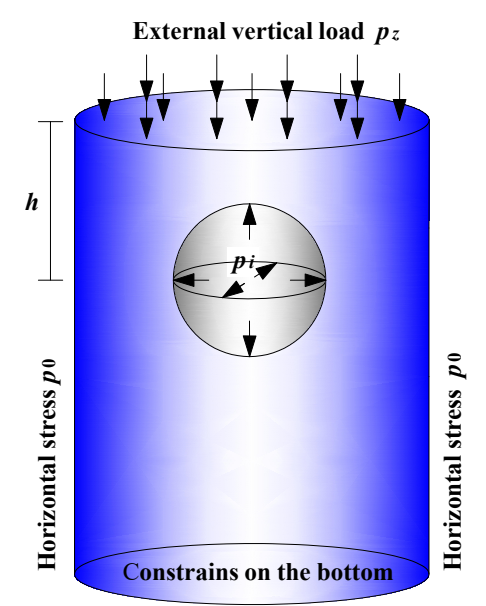

Figure 1 Sketch of limestone strata in a mantled Karst region, containing shallow buried spherical Karst cave with fill material

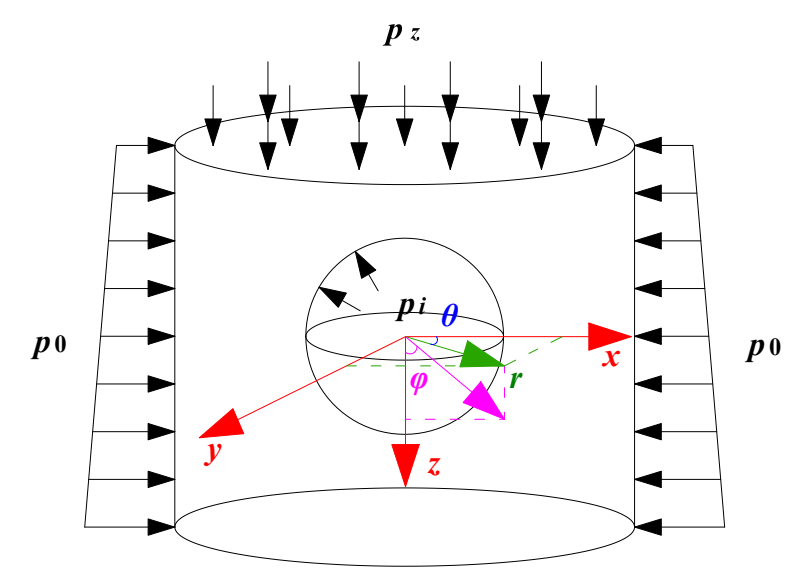

Figure 2 Mechanical model 


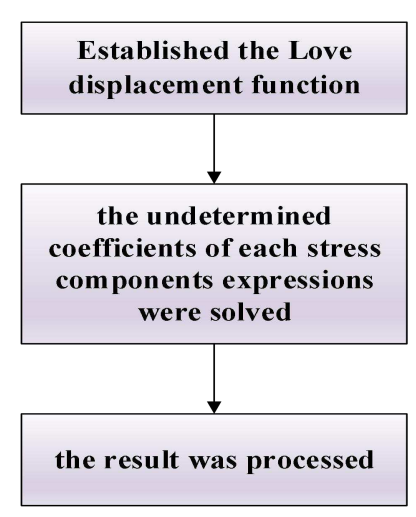

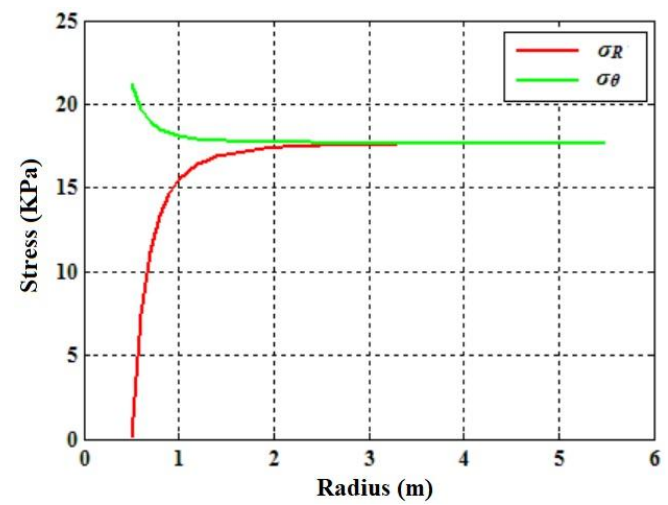

(a)

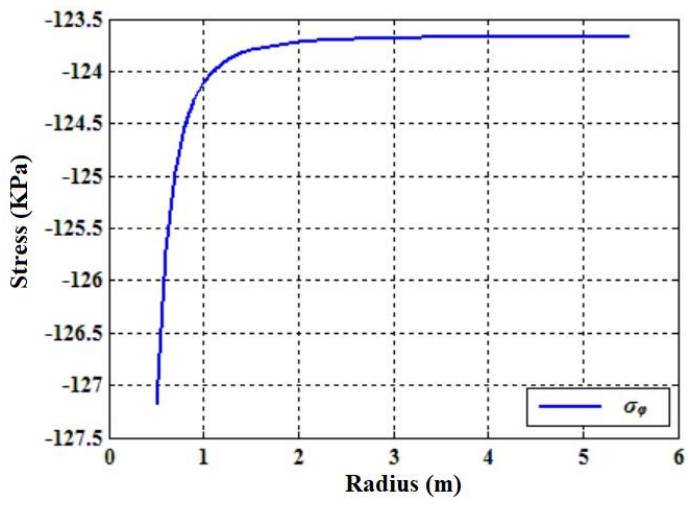

(b)

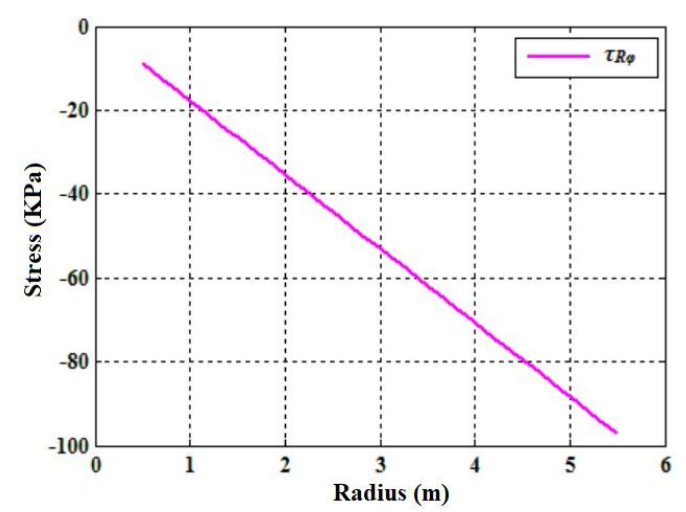

342 Figure 4 The distribution of stress component effected by single influencing factor $((\varphi=\pi / 2$, and radius $(R)$ varies from $0.5 \mathrm{~m}$ to $5.5 \mathrm{~m}$ ) (a) the distribution of circumferential stress and radial stress, (b) the distribution of tangential stress, (c) the distribution of shear stress. 


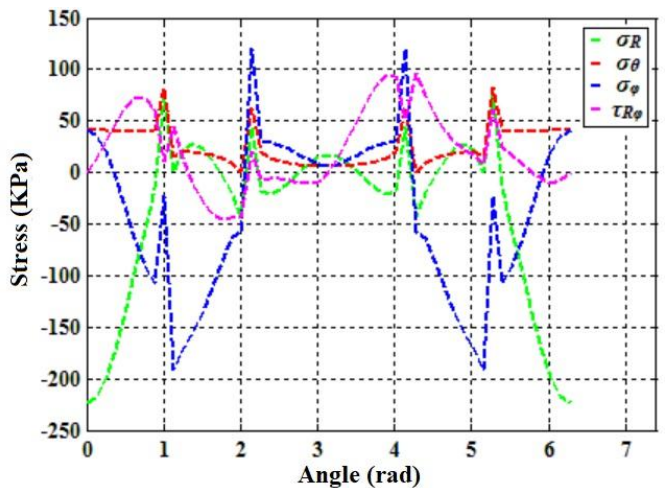

346 Figure 5 The distribution of stress component effected by single influencing factor ((radius $(R)=$ $3472 \mathrm{~m}$, and angle $(\varphi)$ varies from 0 to $2 \pi)$.
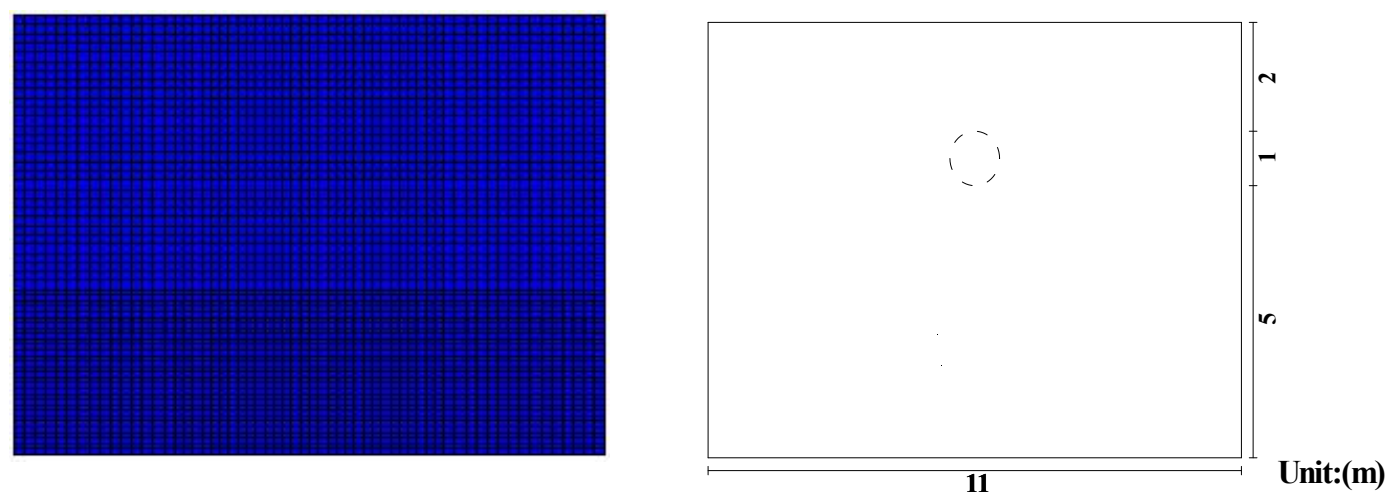

Figure 6 Sketch of model for numerical simulation

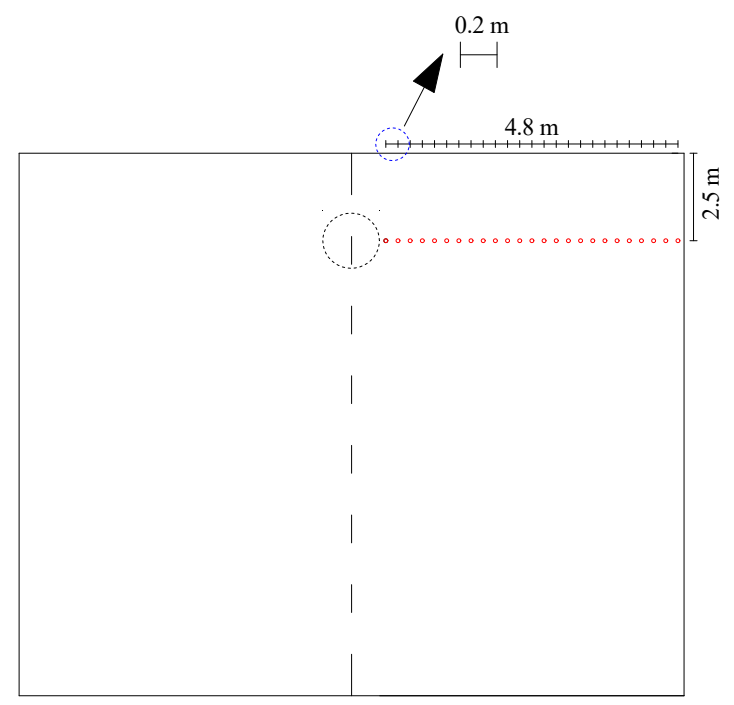




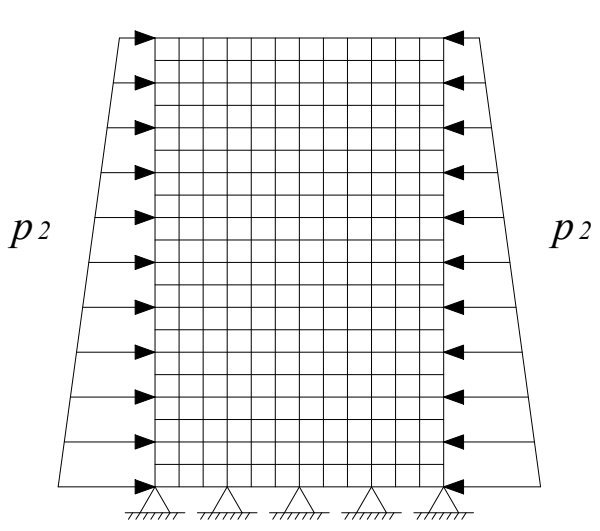

352

353

354

355

356

357

358

Table 1 Parameters of limestone strata

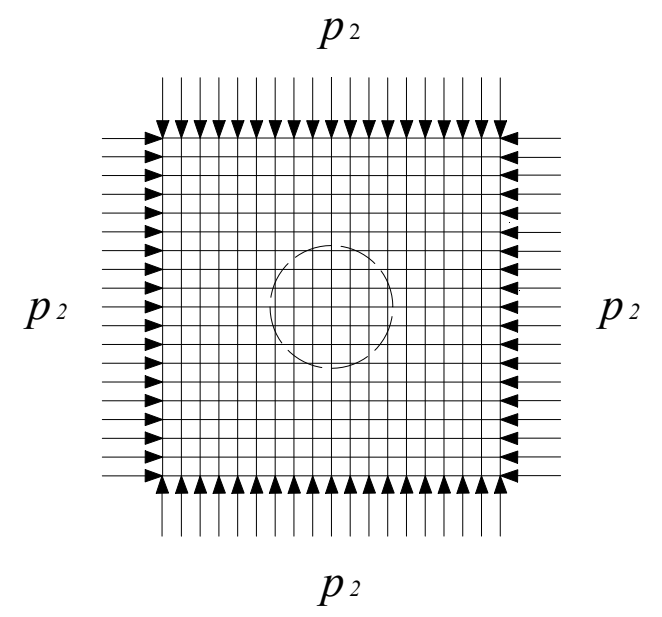

(b)

Figure 8 Sketch of constrained condition and boundary condition for numerical simulation model

(a) vertical cross-section (b) horizontal cross-section

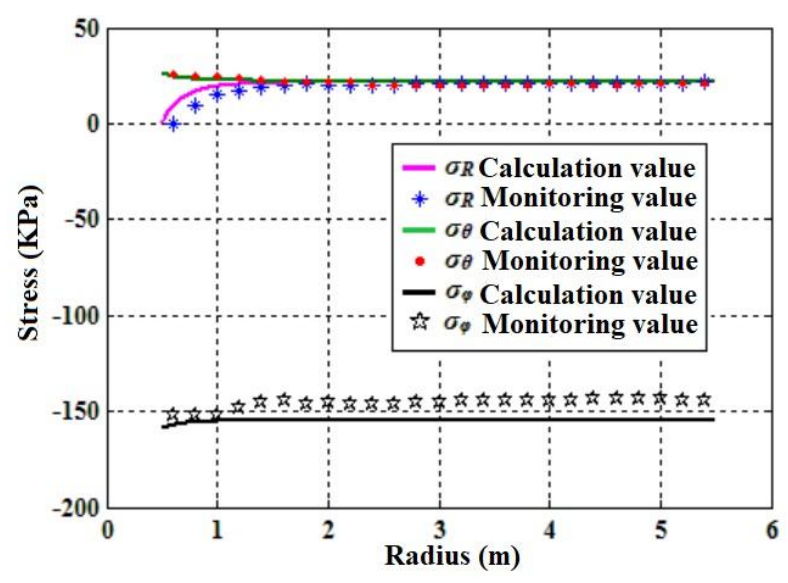

Figure 9 Comparison between theoretical analysis results and numerical simulation results

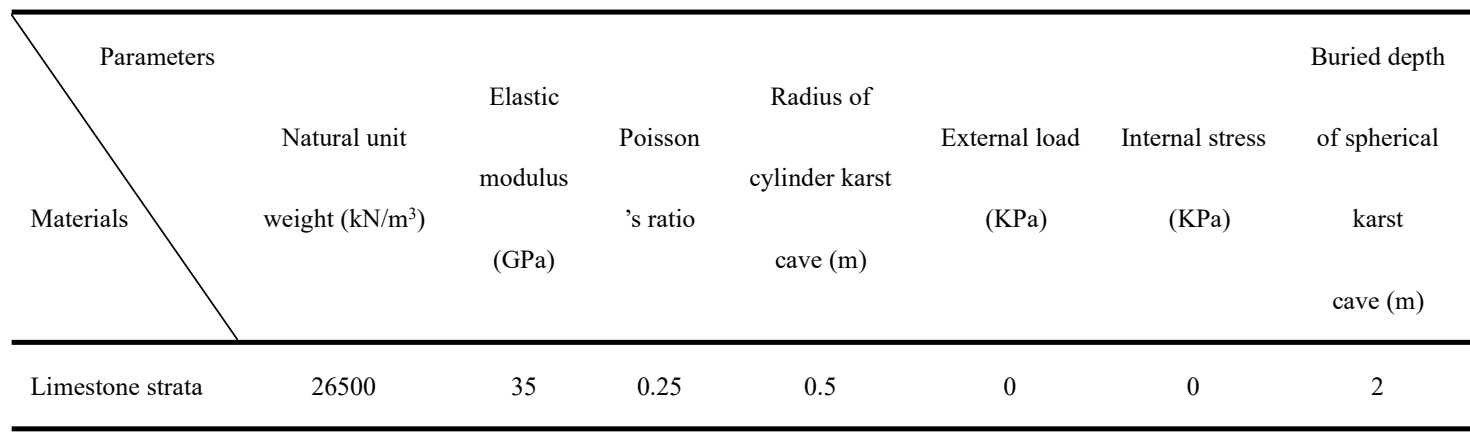

